\title{
A Ripplet Transform Based Statistical Framework for Natural Color Image Retrieval
}

\author{
Manish Chowdhury, Sudeb Das, and Malay K. Kundu \\ Machine Intelligence Unit, Indian Statistical Institute, Kolkata 700 108, India \\ \{st.manishc, to.sudeb\}@gmail.com, malay@isical.ac.in
}

\begin{abstract}
We present a novel Content Based Image Retrieval (CBIR) scheme for natural color images using Multi-scale Geometric Analysis (MGA) of Ripplet Transform (RT) Type-I in the statistical framework based on Generalized Gaussian Density (GGD) model and KullbackLeibler Distance (KLD). The system is based on modeling the marginal distributions of RT coefficients by GGD framework and computing the similarity between the model parameters using the KLD. Least SquareSupport Vector Machine (LS-SVM) classifier is used to classify the images of the database. Extensive experiments were carried out to evaluate the effectiveness of the proposed system on two image databases consisting 1000 (Simplicity) and 2788 (Oliva) images, respectively. Experimental results and comparisons show that the proposed CBIR system performs efficiently in image retrieval field.
\end{abstract}

Keywords: RT, KLD, LS-SVM, CBIR, MGA, GGD.

\section{Introduction}

The task of retrieving relevant images from a large image database (DB), by measuring similarities between the query image and the database images has become a potential area of research. High retrieval efficiency and less computational complexity, are the desired characteristics of an efficient CBIR system [3].

A modern CBIR system consists of three main parts: feature extraction (FE), similarity measurement (SM) and relevance feedback (RF), respectively. Various FE schemes have been reported in the literature for finding the significant visual information of the images [4. Generally, there are two types of image representation: spatial domain representation and frequency domain representation. It has been found that the frequency domain representations are more robust against noise and capable of representing images more efficiently than the spatial domain representation 14 .

Among the various Multiresolution Analysis (MRA) tool, Wavelet Transform (WT) and its variants (like M-band WT, WT packets etc.) have been used in CBIR systems extensively [1410]. But the problem with WT is that it is inherently non-supportive to directionality and anisotropy. To overcome these limitations of WT, recently a theory called Multi-scale Geometric Analysis (MGA) for 
high-dimensional signals has been proposed and several MGA tools are developed like Curvelet, Contourlet etc., with applications in various problems 61. CBIR systems based on these MGA tools are found to perform better than the system based on WT [157]. Recently, Ripplet Transform (RT) Type-I is proposed as an extension to Curvelet Transform (CVT) with alternative scaling law, and possessing higher efficiency for various image processing applications [182]. In frequency domain methods, feature vectors are computed from various simple subbands' statistics (such as mean, standard deviation and energy etc.,) [2. Whereas, in statistical approach, images are modeled in terms of statistical distribution 315. In this article, we have extended this statistical framework to RT for constructing an efficient image representative feature vector.

Many machine learning techniques such as MLP, SVM, etc., have been used to improve the retrieval accuracy of the CBIR system [19]13. However, the training process of MLP is time consuming and its convergence depends on the initial parameter setting. Similarly, training with SVM is computationally expensive for high dimensional data sets, such as image data. To reduce the computational demand, the least square version of SVM, i.e. least square support vector machine (LS-SVM) was developed, which solves in a set of linear equations instead of quadratic programming and simplifies the training procedure [16. Therefore, to reduce the time complexity and prolonged training process for parameters optimization, we have used LS-SVM in our proposed CBIR system.

Traditionally, the most commonly used similarity measures in CBIR is Euclidean distance (ED). ED is often used in CBIR, because of its computational simplicity and rotation invariance property [3]. However, the main problem using $\mathrm{ED}$ is its scale problem, because features that have an inherently larger scale would be predominant. In contrast to these similarity measures, Kullback Leibler Divergence (KLD), an information-theoretic measure is found to be more effective [5].

Even though statistical modeling has been used extensively in texture analysis problem [3,5]. The effectiveness of these approaches in natural color image classification is not explored well. In this article, we propose to apply the statistical framework based on Generalized Gaussian Density (GGD) and KLD on Discrete Ripplet Transform (DRT) coefficients to investigate its performance in natural color image classification problem. To improve the retrieval accuracy as well as to reduce the computational complexity, we have used LS-SVM in the proposed system. Extensive experiments and comparisons with state-of-the-art CBIR systems, shows that the proposed CBIR system works more efficiently .

The paper is organized as follows: Section 2 describes the theoretical preliminary of the RT. The detail descriptions of the feature extraction procedure, classification through LS-SVM and KLD based similarity measure are explained in Section 3, Section 4 and Section 5, respectively. The proposed CBIR system is described in Section 6. Section 7 discusses the experimental system and results. Finally, Section 8 concludes the article. 


\section{Ripplet Transform Type-I (RT)}

Conventional transforms like Fourier Transform (FT) and WT suffer from discontinuities such as edges and contours in images. To address this problem, Jun $\mathrm{Xu}$ et al. proposed a new MGA-tool called RT [18. RT is a higher dimensional generalization of the CVT. RT provides a new tight frame with sparse representation for images with discontinuities along $C^{d}$ curves. There are two questions regarding the scaling law used in CVT: 1) Is the parabolic scaling law optimal for all types of boundaries? and if not, 2) What scaling law will be optimal? To address these questions, Jun $\mathrm{Xu}$ et al., intended to generalize the scaling law, which resulted in RT. RT generalizes CVT by adding two parameters, i.e., support $c$ and degree $d$. CVT is a special case of RT with $c=1$ and $d=2$.

As digital image processing needs discrete transform instead of continuous transform, here we describe the discretization of RT [18. In the frequency domain, the corresponding frequency response of ripplet function is in the form

$$
\widehat{\rho}_{j}(r, \omega)=\frac{1}{\sqrt{c}} a^{\frac{m+n}{2 n}} W\left(2^{-j} \cdot r\right) V\left(\frac{1}{c} \cdot 2^{-\left\lfloor j \frac{m-n}{n}\right\rfloor} \cdot \omega-l\right)
$$

where $\mathrm{W}$ and $\mathrm{V}$ are the 'radial window' and 'angular window', respectively and satisfy the following admissibility conditions:

$$
\begin{gathered}
\sum_{j=0}^{+\infty}\left|W\left(2^{-j} \cdot r\right)\right|^{2}=1 \\
\sum_{l=-\infty}^{+\infty}\left|V\left(\frac{1}{c} \cdot 2^{-\lfloor j(1-1 / d)\rfloor} \cdot \omega-l\right)\right|^{2}=1
\end{gathered}
$$

given $c, d$ and $j$. Here, the scale parameter $a$ is sampled at dyadic intervals. $b$ (position parameter) and $\theta$ (rotation parameter) are sampled at equal-spaced intervals. $a_{j}, \vec{b}_{k}$ and $\theta_{l}$ substitute $a, \vec{b}$ and $\theta$ respectively, and satisfy that $a_{j}=2^{-j}, \vec{b}_{k}=\left[c \cdot 2^{-j} \cdot k_{1}, 2^{-j / d} \cdot k_{2}\right]^{T}$ and $\theta_{l}=\frac{2 \Pi}{c} \cdot 2^{-\lfloor j(1-1 / d)\rfloor} \cdot l$, where $\vec{k}=\left[k_{1}, k_{2}\right]^{T}$, and $j, k_{1}, k_{2}, l \in \mathbb{Z} .(\cdot)^{T}$ denotes the transpose of a vector. $d \in \mathbb{R}$, since any real number can be approximated by rational numbers, so we can represent $d$ with $d=n / m, n, m \neq 0 \in \mathbb{Z}$. Usually, we prefer $n, m \in \mathbf{N}$ and $n, m$ are both primes.

The 'wedge' corresponding to the ripplet function in the frequency domain is

$$
H_{j, l}(r, \theta)=\left\{2^{j} \leq|r| \leq 2^{2 j},\left|\theta-\frac{\pi}{c} \cdot 2^{-\lfloor j(1-1 / d)\rfloor} \cdot l\right| \leq \frac{\pi}{2} 2^{-j}\right\}
$$

The DRT of an $M \times N$ image $f\left(n_{1}, n_{2}\right)$ is in the form of

$$
R_{j, \vec{k}, l}=\sum_{n_{1}=0}^{M-1} \sum_{n_{2}=0}^{N-1} f\left(n_{1}, n_{2}\right) \overline{\rho_{j, \vec{k}, l}\left(n_{1}, n_{2}\right)}
$$

where $R_{j, k}, l$ are the ripplet coefficients and $\overline{(.)}$ denotes the conjugate operator. 


\section{$3 \quad$ Ripplet Coefficient Modeled Using GGD}

The images in the DB prior to RT decomposition are transformed from RGB to $\mathrm{YCbCr}$ color space. This ensures that the textural characterization of the images are independent of the color characterization. RT decomposition over the intensity plane $(\mathrm{Y})$ characterizes the texture information, while the RT decomposition over chromaticity planes $(\mathrm{Cb}$ and $\mathrm{Cr}$ ) characterizes color. Texture and color information are extracted by using RT on each color plane with 4 level $(1,2,4,4)$ decomposition. This decomposition configuration produces 11 $(=1+2+4+4)$ subbands for each image of the DB for each color plane. As, there are 3 color planes, so altogether we get $33(=3 \times 11)$ subbands for each image of the DB. The distribution of ripplet subband coefficients are then modeled with GGD which is defined as,

$$
p(x ; \alpha, \beta)=\frac{\beta}{2 \alpha \Gamma(1 / \beta)} e^{-(|x| / \alpha)^{\beta}}
$$

where $x$ is the ripplet subband coefficients and $\Gamma($.$) is the gamma function, i.e.,$ $\Gamma(z)=\int_{0}^{\infty} e^{-t} t^{z-1} d t, z>0$. Here the scale parameter $\alpha$ models the width of the probability distribution function (PDF) peak (standard deviation), while the shape parameter $\beta$ is inversely proportional to the decreasing rate of the peak. These two parameters need to be estimated for feature vector creation. As Maximum Likelihood (ML) estimator is best suited for estimating heavytailed distribution like GGD for both small and large samples, we have used ML estimator in our proposed scheme.

For the sample set $x=\left(x_{1}, x_{2}, x_{3}, \ldots, x_{k}\right), x_{i}$ is the ripplet coefficients at the $i^{t h}$ subband, and $i \leq L$, the ML estimator is defined as [5].

$$
L(x ; \alpha, \beta)=\log \prod_{i=1}^{L} p\left(x_{i} ; \alpha, \beta\right)
$$

GGD parameters are defined with the following equations, which have a unique root in probability

$$
\begin{gathered}
\frac{\partial L(x ; \alpha, \beta)}{\partial \alpha}=-\frac{L}{\alpha}+\sum_{i=1}^{L} \frac{\beta\left|x_{i}\right|^{\beta} \alpha^{-\beta}}{\alpha}=0 \\
\frac{\partial L(x ; \alpha, \beta)}{\partial \beta}=\frac{L}{\beta}+\frac{L \Psi(1 / \beta)}{\beta^{2}}-\sum_{i=1}^{L}\left(\frac{\left|x_{i}\right|}{\alpha}\right)^{\beta} \log \left(\frac{\left|x_{i}\right|}{\alpha}\right)=0
\end{gathered}
$$

where $\Psi($.$) is the digamma function, i.e., \Psi(z)=\Gamma^{\prime}(z) / \Gamma(z)$. $\alpha$ has a unique, real, positive solution and can be obtained from Eq.(8) by fixing $\beta>0$ :

$$
\hat{\alpha}=\left(\frac{\beta}{L} \sum_{i=1}^{L}\left|x_{i}\right|^{\beta}\right)^{1 / \beta}
$$


Substituting this into (9), the shape parameter $\beta$ is the solution of the following transcendental equation

$$
1+\frac{\Psi(1 / \hat{\beta})}{\hat{\beta}}-\frac{\sum_{i=1}^{L}\left|x_{i}\right|^{\hat{\beta}} \log \left|x_{i}\right|}{\sum\left|x_{i}\right|^{\hat{\beta}}}+\frac{\log \left(\frac{\hat{\beta}}{L} \sum_{i=1}^{L}\left|x_{i}\right|^{\hat{\beta}}\right)^{1 / \hat{\beta}}}{\hat{\beta}}=0
$$

which can be solved numerically using the Newton-Raphson iterative procedure. Therefore, we obtain two features from a ripplet subband $(l)$. Considering $s$ $(=3 l)$ as the total number of subbands for an image $I$, we obtain 2 s dimensional ripplet GGD feature vector. The final feature vector of an image $I$ in the DB is as follows:

$$
f_{v e c}^{I}=\left[\alpha_{1}, \beta_{1}, \alpha_{2}, \beta_{2}, \ldots, \alpha_{s}, \beta_{s},\right]
$$

where $\alpha_{l}$ and $\beta_{l}$ are the GGD parameters computed from the coefficients of the $l^{\text {th }}$ ripplet subband and $1 \leq l \leq s$.

\section{Classification through LS-SVM}

Once the ripplet GGD feature vectors are obtained, LS-SVM classifier is used to classify images of the database. LS-SVM avoids solving quadratic programming problem and simplifies the training procedure of conventional SVM [16]. Considering a linearly separable binary classification problem:

$$
\left(x_{i}, y_{i}\right)_{i=1}^{n} \quad \text { and } \quad y_{i}=\{+1,-1\}
$$

where $x_{i}$ is an n-dimensional vector and $y_{i}$ is the label of this vector. LS-SVM can be formulated as the optimization problem:

$$
\min _{w, b, e} \mathcal{J}(w, b, e)=\frac{1}{2} w^{\prime} w+\frac{1}{2} C \sum_{i=1}^{n} e_{i}^{2}
$$

subject to the equality constraints

$$
y_{i}\left[w^{\prime} \varphi\left(x_{i}\right)+b\right]=1-e_{i}
$$

where $C>0$ is a regularization factor, $b$ is a bias term, $w$ is the weights vector, $e_{i}$ is the difference between the desired output and the actual output and $\varphi\left(x_{i}\right)$ is a mapping function.

The lagrangian for problem of Eq.(14) is defined as follows:

$$
\begin{gathered}
\mathcal{L}\left(w, e_{i}, b, \alpha_{i}\right)=\min _{w, b, e} \mathcal{J}(w, b, e)- \\
\sum_{i=1}^{n} \alpha_{i}\left\{y_{i}\left[w^{\prime} \varphi\left(x_{i}\right)+b\right]-1+e_{i}\right\}
\end{gathered}
$$

where $\alpha_{i}$ are Lagrange multipliers. The Karush-Kuhn-Tucker (KKT) conditions for optimality 
$\frac{\partial \mathcal{L}}{\partial w}=0 \rightarrow w=\sum_{i=1}^{n} \alpha_{i} y_{i} \varphi\left(x_{i}\right) ; \frac{\partial \mathcal{L}}{\partial e_{i}}=0 \rightarrow \alpha_{i}=C e_{i} ; \frac{\partial \mathcal{L}}{\partial b}=0 \rightarrow \sum_{i=1}^{n} \alpha_{i} y_{i}=$ $0 ; \frac{\partial \mathcal{L}}{\partial \alpha_{i}}=0 \rightarrow y_{i}\left[w^{\prime} \varphi\left(x_{i}\right)+b\right]-1+e_{i}=0$,is the solution to the following linear system

$$
\left[\begin{array}{cc}
0 & -Y \\
Y & \varphi \varphi^{\prime}+C^{-1} I
\end{array}\right]\left[\begin{array}{l}
b \\
\alpha
\end{array}\right]=\left[\begin{array}{l}
0 \\
\overline{1}
\end{array}\right]
$$

where $\varphi=\left[\varphi\left(x_{1}\right)^{\prime} y_{1}, \ldots, \varphi\left(x_{n}\right)^{\prime} y_{n}\right], Y=\left[y_{1}, \ldots, y_{n}\right], \overline{1}=[1, \ldots, 1]$, and $\alpha=$ $\left[\alpha_{1}, \ldots, \alpha_{n}\right]$. For a given kernel function $\mathrm{K}($,$) and a new test sample point \mathrm{x}$, the LS-SVM classifier is given by

$$
f(x)=\operatorname{sgn}\left[\sum_{i=1}^{n} \alpha_{i} y_{i} K\left(x, x_{i}\right)+b\right]
$$

\section{$5 \quad$ Similarity Measurement Using KLD}

After the image is classify using LS-SVM according to the ripplet GGD feature vectors, KLD is used for distance calculation between the query image and the database images.

Let $p_{1}(x)$ and $p_{2}(x)$ be two continuous probability distribution functions. KLD distance between these two probability distribution function is defined by [5]

$$
D\left(p_{1}, p_{2}\right)=\int p_{1}(x) \log \left(\frac{p_{1}(x)}{p_{2}(x)}\right) d x
$$

We obtain the KLD between two GGDs substituting the GGD probability distribution function of Eq.(6) into Eq.(19), which is defined as [5]

$$
\begin{array}{r}
D\left(p\left(, ; \alpha_{1}, \beta_{1}\right) \| p\left(, ; \alpha_{2}, \beta_{2}\right)\right)=\log \frac{\beta_{1} \alpha_{2} \Gamma\left(1 / \beta_{2}\right)}{\beta_{2} \alpha_{1} \Gamma\left(1 / \beta_{1}\right)} \\
+\left(\frac{\alpha_{1}}{\alpha_{2}}\right)^{\beta_{2}} \frac{\Gamma\left(\left(\beta_{2}+1\right) / \beta_{1}\right)}{\Gamma\left(1 / \beta_{1}\right)}-\frac{1}{\beta_{1}}
\end{array}
$$

Ripplet coefficients of each subband are assumed to be independent. Therefore, KLD between two images is obtained by calculating the sum of all the Kullback-Leibler distances across all the ripplet subbands of these images. Let, $\alpha_{j}^{i}$ and $\beta_{j}^{i}$ be the ripplet GGD features of subband $j$ of image $i$. Using the definition of KLD between two GGDs shown in Eq. (20), the KLD between two images $\mathrm{P}$ and $\mathrm{Q}$ is defined by Eq. (21)

$$
\begin{array}{r}
D(P, Q)=\sum_{j=1}^{s} D\left(p\left(. ; \alpha_{j}^{(P)}, \beta_{j}^{(P)}\right) \| p\left(. ; \alpha_{j}^{(Q)}, \beta_{j}^{(Q)}\right)\right) \\
=\sum_{j=1}^{s} \log \frac{\beta_{j}^{(P)} \alpha_{j}^{(Q)} \Gamma\left(1 / \Gamma_{j}^{(Q)}\right)}{\beta_{j}^{(Q)} \alpha_{j}^{(P)} \Gamma\left(1 / \Gamma_{j}^{(P)}\right)}+ \\
\sum_{j=1}^{s}\left(\frac{\alpha_{j}^{(P)}}{\alpha_{j}^{(Q)}}\right)^{\beta_{j}^{(Q)}} \frac{\Gamma\left(\left(\beta_{j}^{(Q)}+1\right) / \beta_{j}^{(P)}\right)}{\Gamma\left(1 / \beta_{j}^{(P)}\right)}-\sum_{j=1}^{s} \frac{1}{\beta_{j}^{(P)}}
\end{array}
$$


where $s$ is the total number of ripplet subbands of an image. For a given query image, KLD is measured between the query image and each of the database images using Eq.(21). Database images are ranked according to ascending order of distances and are retrieved from the database.

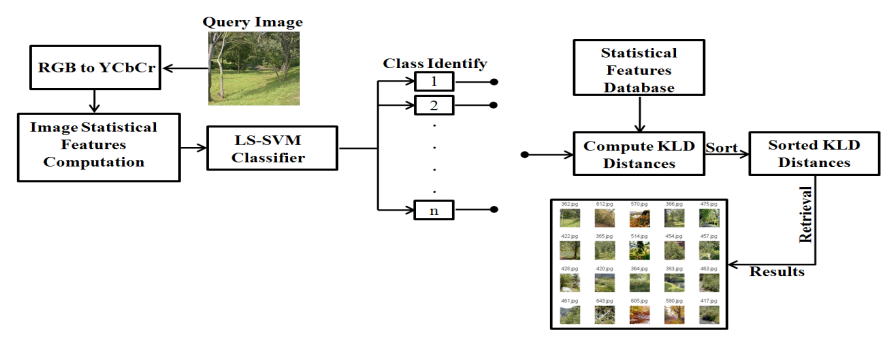

Fig. 1. Block Diagram of the Proposed CBIR System

\section{Proposed CBIR System}

Here, we outlines the salient steps of the proposed CBIR system, considering the statistical features of the images in the database are already computed and stored in the 'Statistical Features Database'. The Fig.1, shows the block diagram of the proposed CBIR system.

\section{Steps:}

1. Input the query image.

2. Convert the query image from RGB color plane to YCbCr color plane.

3. Compute the GGD based statistical parameters of the query image as described in Section 3 .

4. Identify the class of the query image using LS-SVM, depending on the RT based statistical features as described in Section 4.

5. As describe in Section 5. find the KLD distances between the statistical features of the query image and the statistical features of the image DB stored in "Statistical features Database".

6. Sort the distances in ascending order and display 20 images corresponding to the first 20 sorted distances.

7. Stop.

\section{Experimental Results}

Extensive experiments were carried out to evaluate the performance of the proposed CBIR system, and its performance is compared with several state-of-theart CBIR systems. 


\subsection{Experimental Setup}

Two publicly available image databases: (1) SIMPLIcity [17 and (2) Oliva 12 were used in the experiments. SIMPLIcity database consists of 1000 images of 10 different categories containing 100 images each. Oliva dataset consists of 2688 images with 8 categories. LS-SVM classifier is trained with $70 \%$ and tested with $30 \%$ of the leveled data from the image database using stratified random sampling method, respectively. For training, we have used the Radial Basis Function $(\mathrm{RBF})$, as the kernel. There are two tunable parameters while using the RBF kernel in LS-SVM classifier: $\mathrm{C}$ and $\gamma$. It is not known beforehand which values of $\mathrm{C}$ and $\gamma$ are the best for the classification problem at hand. Hence, a $2 X 5$ fold cross-validation $(\mathrm{CV})$ is conducted, where various pairs of $(\mathrm{C}, \gamma)$ are tried and the one with the lowest CV error rate is picked. We have achieved the classification accuracy of $80 \%$ for SIMPLIcity image database. With similar configuration, $75 \%$ the classification accuracy is obtained for Oliva dataset.

Quantitative evaluation of the proposed CBIR system is analyzed using two statistical measures: Mean average precision (MAP) and Mean average recall (MAR). We computed the precisions and recalls considering all the images of the used databases as the query images, and then took the average of the obtained precision and recall values over all the images as the final evaluation result.

\subsection{Results and Discussion}

The retrieval performance of the proposed CBIR system (RT+GGD+LS-SVM) is shown in the Fig. 2, in terms of precision and recall curve. The performance comparisons of WT and CVT with RT are also shown in Fig. 2 (WT+GGD+LSSVM and CVT+GGD+LS-SVM, respectively). Here, only the images of the SIMPLIcity database were considered for performance evaluation. It is clear from the Fig. 2, that RT performance much better than WT and CVT in CBIR domain.

Fig. 3(a) and Fig. 3(b), shows two examples of the visual results obtained by the proposed CBIR system on the SIMPLIcity and Oliva dataset using query images from "Building" and "Forest" classes, respectively. From all the given instances, we can clearly see that all the retrieved images are from the respective classes corresponding to the query images.

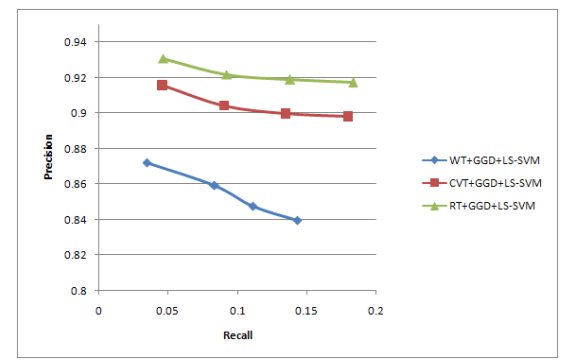

Fig. 2. Performance comparison in terms of precision and recall curves 


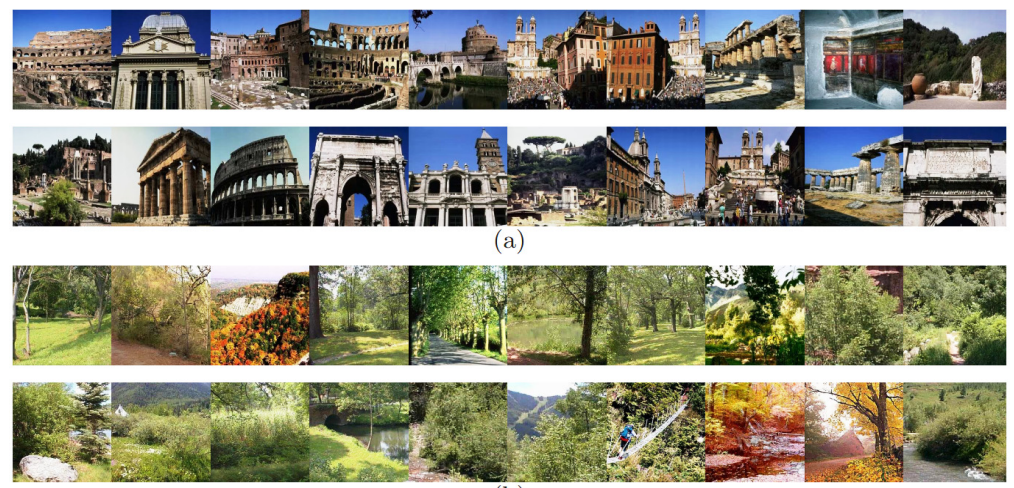

(b)

Fig. 3. Visual results of the proposed CBIR system for SIMPLIcity and Oliva database (top left image is the query image)

Table 1. Comparisons with other existing CBIR systems in terms of average precision on SIMPLicity database

\begin{tabular}{|c|c|c|c|c|}
\hline Class & Wang $[17]$ & CTCHIRS $[1]$ & IGA-CBIR $[9]$ & Proposed Method \\
\hline Africans & 48 & 68.30 & 61 & $\mathbf{8 9 . 4 2}$ \\
\hline Sea & 32 & 54 & $\mathbf{9 3}$ & 76.66 \\
\hline Building & 35 & 56.15 & 85 & $\mathbf{8 6 . 0 4}$ \\
\hline Buses & 36 & 88.80 & 71 & $\mathbf{9 7 . 9 0}$ \\
\hline Dinosaurs & 95 & 99.25 & $\mathbf{1 0 0}$ & $\mathbf{1 0 0}$ \\
\hline Elephant & 38 & 65.80 & 80 & $\mathbf{9 3 . 7 7}$ \\
\hline Flower & 42 & 89.10 & 82 & $\mathbf{9 8 . 0 8}$ \\
\hline Horse & 72 & 80.25 & 79 & $\mathbf{1 0 0}$ \\
\hline Mountain & 35 & 52.15 & 56 & $\mathbf{8 5 . 9 6}$ \\
\hline Food & 38 & 73.25 & $\mathbf{9 9}$ & 96.22 \\
\hline Average & 47 & 72.70 & 80.6 & $\mathbf{9 2 . 4 0}$ \\
\hline
\end{tabular}

Table 1, shows the results of the performance comparisons between the proposed CBIR system with some of the existing CBIR systems namely, SIMPLIcity 17, CTCHIRS 11 and IGA-CBIR 9. The images of the SIMPLIcity database are used in this experiment. It can be easily seen from the Table 1 that our proposed CBIR system have overall higher retrieval accuracy than these existing systems. It is also to be noted that for only two categories of images ("Sea" and "Food"), the proposed CBIR system have achieved lower retrieval accuracy than the method described in [9]. The 'bold' values of the Table 1 denote the highest retrieval accuracy obtained. The CBIR systems described in [1] and [9] used 71 and 86 features, whereas we have used only 66 features to represent every image of the database. It is obvious from the results given in Table 1 that 
the proposed CBIR system performs much better than the above mentioned existing CBIR schemes. The MAP and MAR of our proposed algorithm is $92.40 \%$ and $16.40 \%$ for SIMPLIcity dataset and for Oliva dataset, $85.98 \%$ and $12.45 \%$ respectively.

\section{Conclusions}

From our experiments, we have noticed that statistical modeling of RT based image coding is suitable for representing low level features of the images. The proposed CBIR system with LS-SVM classifier based on RT based features is able to improve the accuracy of the retrieval performance and to reduce the computational cost. To overcome the problem of misclassification in pre-classification phase, we are trying to implement fuzzy ranking membership function. The proposed mechanism could be tested for video retrieval as future scope of research.

\section{References}

1. Candes, E., Donoho, D.: Continuous curvelet transform: I. resolution of the wavefront set. Appl. and Comput. Harmon. Anal. 19, 162-197 (2003)

2. Chowdhury, M., Das, S., Kundu, M.K.: Novel CBIR system based on ripplet transform using interactive neuro-fuzzy technique. Elect. Letters on Comp. Vision and Image Anal. 11, 1-13 (2012)

3. Datta, R., Joshi, D., Li, J., Wang, J.Z.: Image retrieval: Ideas, influences, and trends of the new age. ACM Comput. Surv. 40, 1-60 (2008)

4. Deselaers, T., Keysers, D., Ney, H.: Features for image retrieval: an experimental comparison. Inform. Retri. 11, 77-107 (2008)

5. Do, M.N., Vetterli, M.: Wavelet-based texture retrieval using generalized gaussian density and kullback-leibler distance. IEEE T. Image Process 11(2), 146-158 (2002)

6. Do, M.N., Vetterli, M.: The contourlet transform: an efficient directional multiresolution image representation. IEEE T. Image Process 14, 2091-2106 (2005)

7. Duc, H.N., Tien, T.L., Honl, T.D., Thu, C.B., Xuan, T.N.: Image retrieval using contourlet based interest points. In: Proc. of the 10th ISSPA, pp. 93-96 (2010)

8. Johnson, D.H., Sinanovic, S.: Symmetrizing the kullback-leibler distance. IEEE T. Inf. Theory (2001)

9. Lai, C.C., Chen, Y.C.: A user-oriented image retrieval system based on interactive genetic algorithm. IEEE T. Instrum. Meas. 60, 3318-3325 (2011)

10. Li, L.Y., Yan, Y., Yang, C.: Implementation of texture based image retrieval using M-band wavelet transform. Wuhan University J. of Natural Sciences 8(4), 11071110 (2003)

11. Lin, C.H., Chen, R.T., Chan, Y.K.: A smart content-based image retrieval system based on color and texture feature. Image Vision Comput. 27, 658-665 (2009)

12. Oliva, A., Torralba, A.: Modeling the shape of the scene: A holistic representation of the spatial envelope. Int. J. of Comp. Vision 42, 145-175 (2001)

13. Pourghassem, H., Ghassemian, H.: Content-based medical image classification using a new hierarchical merging scheme. Comp. Med. Imaging and Graphics 32, 651-661 (2008) 
14. Quellec, G., Lamard, M., Cazuguel, G., Cochener, B., Roux, C.: Adaptive nonseparable wavelet transform via lifting and its application to content-based image retrieval. IEEE T. Image Process 19, 25-35 (2010)

15. Sumana, I.J., Islam, M.M., Zhang, D., Lu, G.: Content based image retrieval using curvelet transform. In: Proc. of 10th IEEE MMSP, pp. 11-16 (2008)

16. Suykens, J.A.K., Vandewalle, J.: Least squares support vector machine classifiers. Neural Process. Lett. 9, 293-300 (1999)

17. Wang, J.Z., Li, J., Wiederhold, G.: SIMPLIcity: Semantics-sensitive integrated matching for picture libraries. IEEE T. Pattern Anal. 23, 947-963 (2001)

18. Xu, J., Yang, L., Wu, D.: Ripplet: A new transform for image processing. J. Vis. Commun. Image R. 21, 627-639 (2010)

19. Yang, C.C., Prashar, S.O., Landry, J.A., Perret, J., Ramaswamy, H.S.: Recognition of weeds with image processing and their use with fuzzy logic for precision farming. Can. Agr. Eng. 42, 195-200 (2000) 